\title{
MULTI-CAMERA LAPAROSCOPIC IMAGING WITH TUNABLE FOCUSING CAPABILITY
}

\author{
A. Kanhere, K. Van Grinsven, C.-C. Huang, Y.-S. Lu, J. Greenberg, C. Heise, Y. Hu and H. Jiang* \\ University of Wisconsin-Madison, USA
}

\begin{abstract}
This paper presents a comprehensive solution to overcome many of the fundamental challenges faced by current laparoscopic imaging systems. Our design features multiple tunable-focus microcameras integrated with a surgical port to provide panoramic intra-abdominal visualization with enhanced depth perception. Our system can be optically tuned to focus and zoom in on objects within a range of $3 \mathrm{~mm}$ to $\infty$, with a field of view adjustable between $36^{\circ}$ and $130^{\circ}$. Our unique approach also eliminates the requirement of an exclusive imaging port and need for navigation of cameras between ports during surgery.
\end{abstract}

\section{INTRODUCTION}

Laparoscopy is a minimally invasive surgical procedure performed through multiple small incisions in the abdomen. These incisions are used to deploy surgical 'trocars' or ports used to introduce cameras and surgical tools inside a surgical cavity. Current laparoscopic imaging technology relies on a single fixedfocus camera through a port, thus facing five fundamental challenges - limited field of view, lack of depth perception, occupation of a dedicated port by the camera, need for extensive maneuvering of the camera during surgery and glare from highly reflective organ surfaces [1].

A panoramic, 3D vision of the surgical field closely resembling the view in a conventional open surgery, is highly desirable in laparoscopy. It helps expedite surgical tasks and enhances the safety of surgical procedures [2]. Research solutions to address these challenges with software techniques for 3-D depth recovery have had limited success [3]. Some commercial systems claim 3D vision by employing a pair of closely spaced cameras. However, the narrow baseline between the cameras and the resulting low disparity limit the accuracy of depth recovery. More importantly, a two-camera pair cannot provide sufficient viewing angles to ensure an unobstructed view of the surgical region despite the presence of surgical instruments.

Current laparoscopes demand one port exclusively for imaging rather than instrumentation. In addition, constant maneuvering of the camera between ports is required to adjust the viewing angle of the surgical field. Several groups have bypassed these requirements by means of imaging tools tethered [4], sutured [5] or magnetically anchored [6] to the abdominal wall. However, these invasive solutions result in additional puncture points or tugging of the abdominal wall, thus diminishing the most appealing benefits of laparoscopy over open surgery.

Existing imaging solutions only address the aforementioned challenges individually and offer partial benefits over the current technology, at best. We previously reported on a reconfigurable fixed-focus, micro-camera array with panoramic vision [7]. Here we present a new comprehensive approach to address the aforementioned challenges in one system.

\section{MECHANISM AND DESIGN}

Our proposed design features an integrated trocar-camera assembly (TCA). The TCA is designed so as to be useful in both single-port and multi-port laparoscopic surgeries. Our TCA integrates an array of microcameras with a surgical port so that multiple cameras may be deployed into the abdominal cavity through an incision, without any additional surgical operation. In addition, it is affixed to the distal end of the port to capture images through simple mechanical actuation without the need for manual intervention.

The TCA consists of four microcameras assembled symmetrically around the trocar, connected together by a mechanical structure. The mechanical design of the assembly is similar to an umbrella frame. As shown in Figure 1, the assembly consists of four mechanical arms, each supporting one tunable microcamera. The arms are interconnected via a mechanical assembly that enables opening and closing of the arms, similar to the spokes of an umbrella. The frame is mounted along the periphery of the distal end of a surgical port, thereby keeping the port accessible. This ensures that the imaging system never interferes with the instruments in the port. The design of the actuation frame enables the reconfiguration of the cameras to ensure complete coverage of the surgical scene without the need for constant maneuvering during surgery.

Images acquired from individual cameras in any one frame position will represent distinct yet overlapping viewing perspectives of the same scene. Hence they can be stitched seamlessly for a much wider field of view in the resulting panoramic image. The use of multiple cameras allows for large field-of-view with reduced aberration (e.g. compared with fisheyelens) while the optical tunability of the microcameras significantly improves the quality and depth perception of the image.

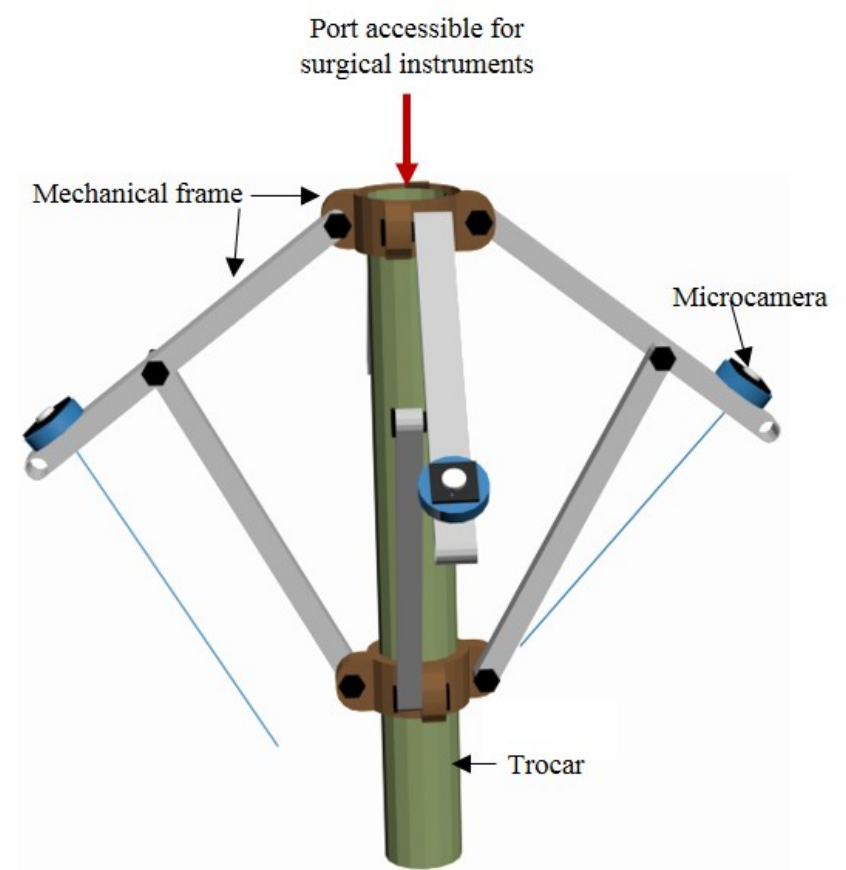

Figure 1: Schematic of the mechanical frame assembly for reconfiguration of camera positions. (Drawing not shown to scale) This design overcomes the problem of dedicated port occupation by imaging tools and keeps all ports accessible for surgical instruments throughout the surgery. 
Each camera unit consists of a $1 \mathrm{~mm} \times 1 \mathrm{~mm}$ NanEye commercial CMOS sensor (AWAIBA Lda, Madeira, Portugal) aligned with a thermo-responsive tunable liquid lens, integrated with a microheater at the base and polarizer in front of the lens.

\section{Variable-focus liquid microlenses}

Since the microcameras will be affixed to the port, they must possess tunable focus in order to provide focused images of a surgical scene. Given the dimensions of an abdominal cavity and the positioning of the cameras closer to the abdominal wall, the tuning range of the focal length was designed to range from a few $\mathrm{mm}$ to $\infty$.

The tuning of focus was achieved by thermo-responsive hydrogel actuation $[8,9]$. The principle of operation of the tunable lens is represented in the schematic shown in Figure 2. The design consists of a thermal stimuli-responsive hydrogel ring placed within a microfluidic chamber filled with water. This chamber is sealed with an aperture slip with a circular opening centered over the ring. An oil chamber is then placed on top of the aperture slip. The opening in the aperture slip acts as the lens aperture. The water-oil meniscus is pinned along the lens aperture by surface treating the layers to create a hydrophobic-hydrophilic contact boundary. The initial meniscus, and thus the focal length, is determined by the volume of water filled in the water chamber. When the temperature of the lens structure is increased, the hydrogel ring responds by contracting due to the release of water via the hydrogel network interstitials. This results in a net change in the volume of the water droplet located in the center of the ring. Since the aperture rim of the lens is pinned, this translates to an increase in the radius of curvature and a decrease in the focal length. This process is reversible, which means that a decrease in temperature will cause the focal length to increase.

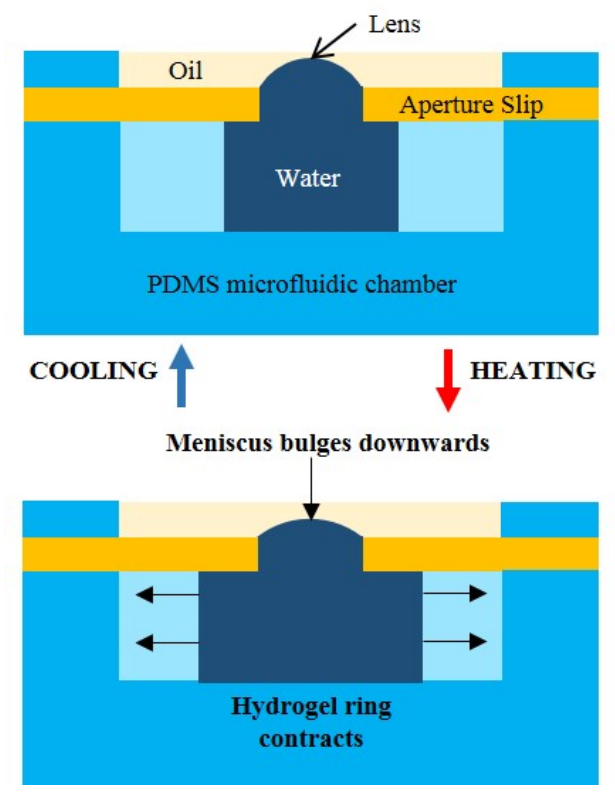

Figure 2: Schematic representation illustrating the operation of a thermo-responsive tunable liquid lens. As the local temperature increases, the hydrogel releases water and contracts, thereby increasing the radius of curvature and decreasing the focal length of the water-oil lens. This focal length change is reversible.

\section{Microheater for focus tuning}

To tune the thermo-responsive hydrogel lens, a microheater was placed at the base of the lens. The temperature range required to tune the microlenses within the desired focal length range of $3 \mathrm{~mm}$ to $\infty$ is $25-60{ }^{\circ} \mathrm{C}$. The factors influencing our choice of the microheater were (1) form factor suitable for easy integration with lenses, (2) uniformity of heat distribution and (3) ease of heat dissipation after operation. We chose a coil type resistive heater design for easy alignment with the hydrogel ring structure. The coil design also minimizes hotspots due to absence of sharp corners [10].

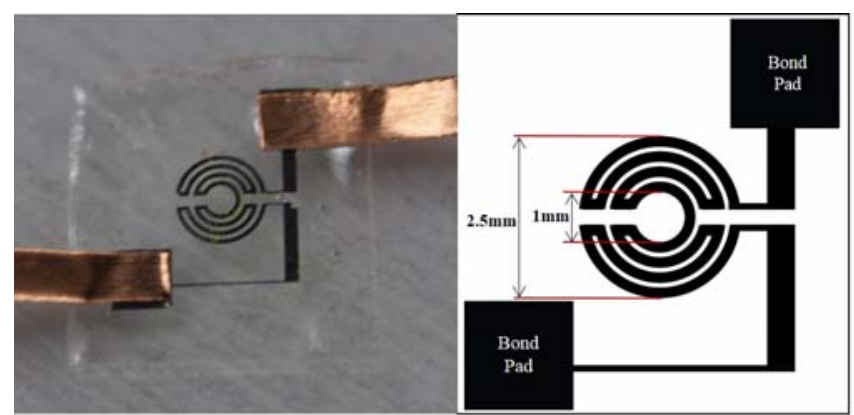

Figure 3 : Image of an aluminum micro heater coil used for tuning the microlenses. Conductive copper tape was used to probe the electrodes.

\section{Polarizer}

The presence of bodily fluids in the body makes the internal organ surfaces highly reflective, resulting in a glare while imaging them. Glossy or highly reflecting surfaces often reflect a linearly polarized, component of the incoming light much more strongly than the others. Therefore, our approach to addressing this challenge was to integrate a polarizer in front of our tunable-focus lens. We fabricated a linear polarizer oriented so as to block the reflected linear component. Such selective transmission of incident light based on the plane of polarization enhances the contrast of objects against the background, especially in low light conditions such as a surgical cavity. The effective reduction of glare with the placement of a polarizer in front of our camera can be seen in Figure 4.
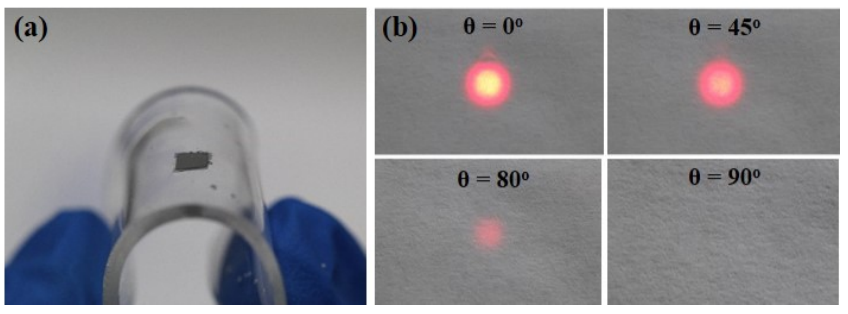

Figure 4: (a) Image of a thin film Polyvinyl Alcohol (PVA) polarizer patterned on a flexible PDMS substrate. (b) Selective transmission of a linearly polarized He-Ne laser $(\lambda=630 \mathrm{~nm})$ when passed through the polarizer.

\section{FABRICATION METHODS}

The tunable lenses were fabricated using liquid-phase photopolymerisation $\left(\mathrm{LP}^{3}\right)$. A 300 -mm-thick cavity was defined on a glass substrate using double-sided adhesive spacers. This cavity was filled with a poly(isobornyl acrylate) (IBA) prepolymer mixture. Poly-IBA posts defining the water container $(\varnothing=2.5 \mathrm{~mm})$ were photopatterned through a photomask aligned on top of the cavity under UV radiance $\left(8.0 \mathrm{~mW} \mathrm{~cm}^{-2}\right.$ for $\left.75 \mathrm{~s}\right)$. The chamber was then rinsed with ethanol to remove the unexposed solution. 
Another $200 \mu \mathrm{m}$ thick chamber was created on top of this structure to define the base of the water container. Then the chamber was filled with pre-cured polydimethylsiloxane (PDMS) and cured at $70{ }^{\circ} \mathrm{C}$ for $8 \mathrm{~h}$, before being peeled off the poly-IBA mold. The sidewalls of the PDMS water containers were treated to be hydrophilic by corona discharge plasma. The water containers were filled then with the N-isopropylacrylamide (NIPAAm) hydrogel pre-polymer solution and then were photopatterned under $\mathrm{UV}$ radiance $\left(\mathrm{I}=15 \mathrm{~mW} \mathrm{~cm} \mathrm{c}^{-2} ; \mathrm{t}=18 \mathrm{~s}\right)$ to form hydrogel rings in the containers.

Similar procedures were carried out to form a $250 \mu \mathrm{m}$ thick PDMS with $1 \mathrm{~mm}$ circular openings defining the lens aperture. The side walls of the aperture were surface treated to be hydrophilic so as to form a pinning boundary for the water-oil lens. Then another 250 um thick PDMS layer with a $2.5 \mathrm{~mm}$ aperture was bonded on top of the aperture slip to form the oil container. The containers were filled with deionized water and silicon oil consecutively, forming the immiscible water-oil interfaces for the microlenses. The cross section of this fabrication process is shown in Figure 2.

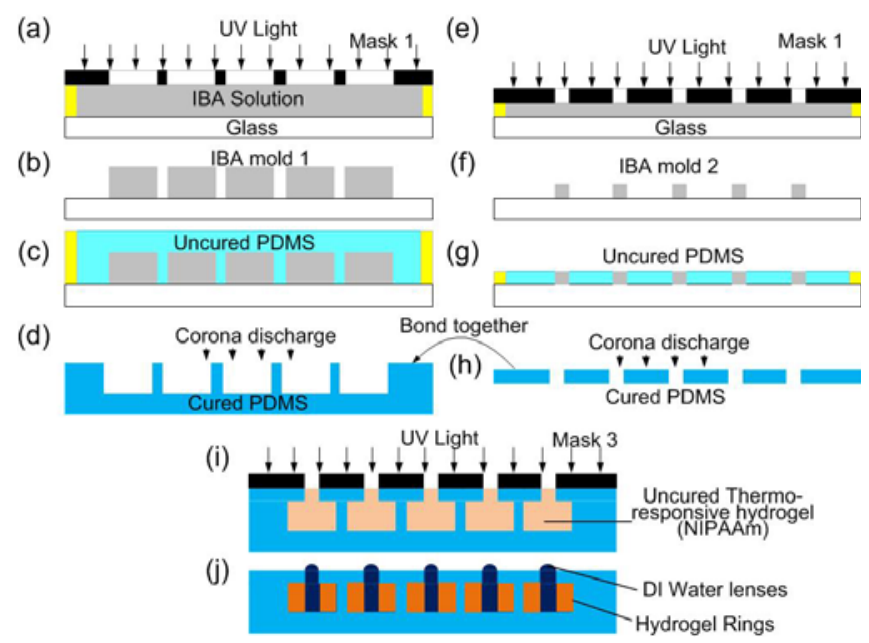

Figure 5: Fabrication process flow for thermo-responsive hydrogel based tunable liquid lenses.

Thermal actuation and tuning of the lens was achieved with a microheater placed at the base of the lens. To tune the thermoresponsive hydrogel lens, a microheater coil was fabricated using a lift-off process and glued to the base of each lens. As shown in Figure 2(b), a layer of PDMS was first spun onto a photoresist coated glass slide and cured at $70^{\circ} \mathrm{C}$ for 4 hours. Subsequently, a 2 um- thick layer of Parylene-C was deposited onto the PDMS layer, to improve the adhesion of metal onto PDMS. A $250 \mathrm{~nm}$ thick Aluminium layer was then sputtered onto this layer followed by photolithography and wet etching $\left(\mathrm{H}_{3} \mathrm{PO}_{4}: \mathrm{HNO}_{3}: \mathrm{CH}_{3} \mathrm{COOH}: \mathrm{H}_{2} \mathrm{O}\right.$ in ratio $3: 3: 1: 1$ at $40^{\circ} \mathrm{C}$ for $75 \mathrm{~s}$ ) to define the microheater coil. The fabrication process for the microheater is demonstrated in Figure 6.

A thin film PVA polarizer was fabricated on a flexible substrate and integrated in front of the camera. First, PDMS elastomer was mixed with a curing agent in 10:1 ratio by weight. The mixture was then placed in the vacuum chamber to extract air bubbles. It was then poured onto a glass slide, cured at $80^{\circ} \mathrm{C}$ for 4 hours and then peeled off to form a flexible PDMS substrate. Then, a pre-polymer of doped Polyvinyl Alcohol (PVA) was spin-coated at $1500 \mathrm{rpm}$ for 1 minute onto the PDMS substrate. The surface was heated at $100^{\circ} \mathrm{C}$ for 20 minutes to evaporate the solvent and cure the PVA thin film.

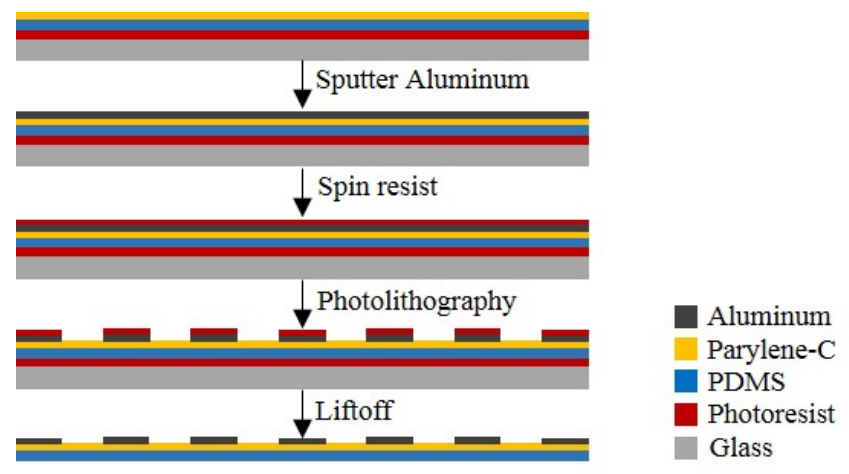

Figure 6: Fabrication process flow for aluminum micro heater coil patterned on PDMS using lift-off process.

\section{RESULTS AND DISCUSSION}

To simulate the surgical environment for our experiments, a test scene was modeled closely on a commonly used scene in laparoscopic training boxes, designed to develop laparoscopic skills in novice surgeons [11]. The test scene was placed in a dark environment with illumination provided solely with a fiber optic light guide to closely resemble a surgical cavity. Since the NanEye image sensors do not have an IR filter, an IR cut was placed at $680 \mathrm{~nm}$ to filter the incident light for faithful color imaging.

To test the optical zoom and focusing capabilities of our cameras, the position of a single tunable camera was fixed $12 \mathrm{~cm}$ away from the test scene. Images of the scene were then captured by the camera while varying the temperature from $25^{\circ} \mathrm{C}$ to $45^{\circ} \mathrm{C}$ to tune the lens. Figure 7 shows a series of three images captured by a camera while tuning the thermo-responsive lens using a microheater. The scene is first out of focus, then the camera gradually focuses and zooms in on the scene as the lens is tuned.

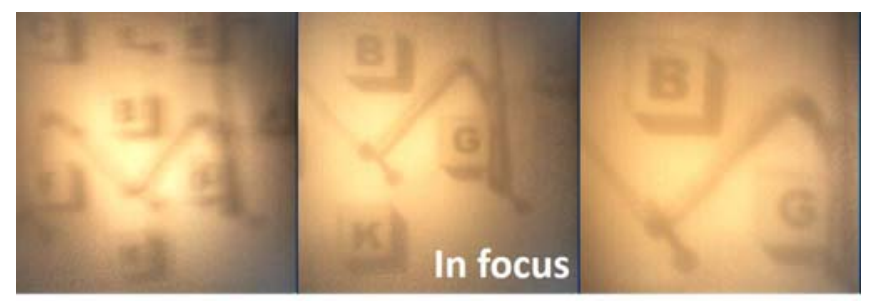

Figure 7 : Images of a test scene as captured by a single camera while the focal length of its tunable liquid lens was being tuned using a microheater.

The effects of integration of a polarizer in front of the camera while imaging reflective surfaces were tested by placing a highly reflective metal nail in the center of the scene being imaged. Figure 8 demonstrates effective glare reduction while imaging with the use of a polarizer.

To test the implementation of our imaging setup in the mock surgical scene, the test scene was sealed within a box with a small opening, similar to an incision in a surgical cavity. The TCA assembly was introduced into the scene along with a fiber optic light guide for illumination. During insertion, the mechanical arms (with the microcameras) were in a closed configuration with the arms parallel to the sidewalls of the port. Once the surgical port was positioned, the arms of the frame were flared out. Initially, the orientation angle of the array was adjusted to provide the desired viewing perspective, depth of field and horizontal field of view. Finer adjustments were then made to ensure distinct but slightly overlapping sub-scenes, as seen by individual cameras. In our 
design, the orientation angle of all the microcameras can be simultaneously adjusted within an error margin of $5^{\circ}$.

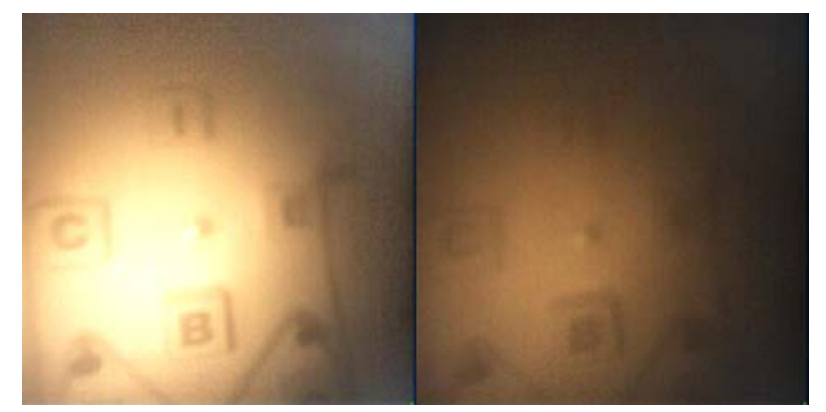

Figure $8:$ Reduction in glare with the use of a polarizer as seen from comparison of an image without using a polarizer in front of a tunable camera (left) and the same image captured with a polarizer in front of the lens (right).

First, the camera lenses were tuned individually to obtain focused images of each of their sub-scenes. This independent optical tuning feature enables our imaging system to handle different object distances without affecting the overall depth perception of the scene. So, sharp images of all the sub scenes are obtained by individual cameras as seen in Figure 9(a-d). These scenes were then stitched together in a commercial software, PTGui to obtain a panoramic image, shown in Figure 9(e). As seen from Figure 9(e), this yields to enhanced depth perception and a significantly large field of view of $128^{\circ}$, which is greater than that of any of the individual source images.

Image distortion can be observed in the panorama in Figure 9(e) that is not seen in any of the sub-scene source images. This is primarily due to limitations of the commercial software. Most commercial softwares have stringent requirements of significant overlap of adjacent images, constant intensity throughout the scene and between successive frames, and identical exposures throughout the scene. However, our device is designed to operate in a surgical, low-light environment where these requirements cannot be met.

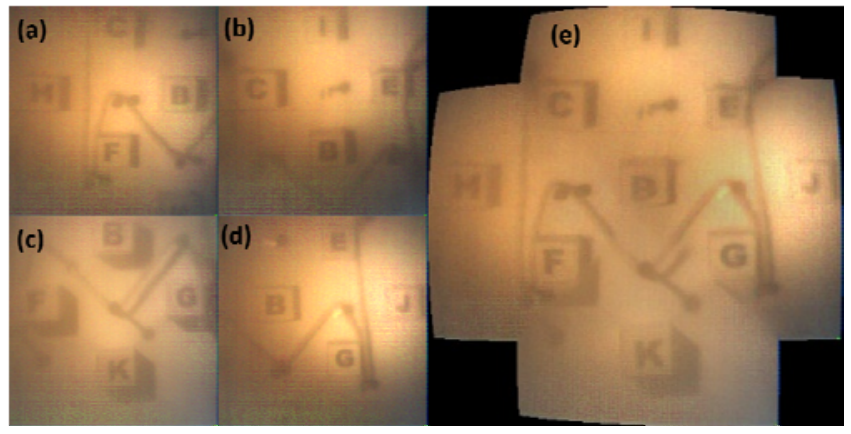

Figure 9: Images of a mimicked surgical scene as captured by our camera array. (a-d) Sharp images of all the sub scenes were obtained by individual cameras These scenes were then stitched together in a commercial software, PTGui to obtain a very large field of view compared to any single camera alone (e).

\section{CONCLUSION}

A multi-camera laparoscopic imaging tool with optical tunability has been demonstrated in this work. Our preliminary results show that our approach offers promising solutions to several challenges including limited field of view and depth perception, need for exclusive imaging ports and extensive maneuvering of the camera during surgery and glare from highly reflective organ surfaces. Our future goals include wireless data transfer, algorithm development for 3D reconstruction, unified user interface design and commercial-grade packaging.

\section{ACKNOWLEDGMENT}

This work was supported by the U.S. National Science Foundation through the Emerging Frontiers in Research and Innovation program (EFRI 0937847). The authors would like to thank Dr. Li Zhang for insightful technical discussions and guidance. We would also like to thank the Transducer Research Foundation for their generous travel support.

\section{REFERENCES}

[1] H. Xin, J.S. Zelek, and H. Carnahan, "Laparoscopic surgery, perceptual limitations and force: A review", First Canadian Student Conference on Biomedical Computing, Kingston, Ontario, Canada, 144 (2006).

[2] Y. Naya, K. Nakamura, K. Araki, K. Kawamura, S. Kamijima, T. Imamoto, N. Nihei, H. Suzuki, T. Ichikawa, and T. Igarashi, "Usefulness of panoramic views for novice surgeons doing retroperitoneal laparoscopic nephrectomy", International Journal of Urology, 16(2008), pp. 177-80.

[3] D. Stoyanov, A. Darzi, and G. Z. Yang, "A Practical Approach towards Accurate Dense 3D Depth Recovery for Robotic Laparoscopic Surgery", Comput. Aided Surg., 10(2005), pp. 199-208.

[4] C.A. Castro, A. Alqassis, S. Smith, T. Ketterl, S. Yu, S. Ross, A. Rosemurg, P.P. Savage, and R.D. Gitlin, "A Wireless Robot for Networked Laparoscopy", IEEE Transactions on Biomedical Engineering, 60(2013), pp. 930-936.

[5] H. Tie, P.K. Allen, T. Nadkarni, N.J. Hogle, and D.L. Fowler, "Insertable stereoscopic 3D surgical imaging device with pan and tilt", IEEE Int. Conf. on Biomedical Robotics and Biomechatronics, Oct 19-22, Scottsdale(2008), pp. $311-316$.

[6] S.L. Best, and J.A. Cadeddu, "Development of magnetic anchoring and guidance systems for minimally invasive surgery", Indian journal of urology, 26(2010), pp. 418-22.

[7] A. Kanhere, B. Aldalali, J. A. Greenberg, C. P. Heise, L. Zhang, and H. Jiang, "Reconfigurable Micro-Camera Array with Panoramic Vision for Surgical Imaging", Journal of Microelectromechanical Systems, 22(2013), pp. 989-991.

[8] L. Dong, A. K. Agarwal, D. J. Beebe and H. Jiang, "Adaptive liquid microlenses activated by stimuli-responsive hydrogels," Nature, 442(2006), pp. 551-554.

[9] X. Zeng*, C. Li*, D. Zhu, H. Cho, and H. Jiang, "Tunable microlens arrays actuated by various thermo-responsive hydrogel structures," IOP Journal of Micromechanics and Microengineering, 20(2010).

[10] C.F. Chau, and T. Melvin, "Design and fabrication of a quasiordered nanoporous silicon membrane suitable for thermally induced drug release", IOP Journal of Micromechanics and Microengineering, 22(2012).

[11] E. Hiemstra, W. Kolkman, M.A.J. van de Put, and F. W. Jansen, "Retention of basic laparoscopic skills after a structured training program", Gynecol Surg., 6(2009), pp. 229-235.

\section{CONTACT}

*H.Jiang, tel: +1-608-265-9418; hongrui@engr.wisc.edu 